\title{
The Challenge of Obesity Management in Primary Care
}

Obesity is increasingly recognized as both a chronic disease for the individual and an epidemic of alarming proportions for our population. Medical concern about obesity does not relate to attractiveness; rather, it focuses on the health impact of excess body weight. Considerable evidence now supports the fear that excess body weight is associated with diminished likelihood of longevity and seriously increases morbidity during life. ${ }^{1}$ Thus far, however, dangers posed by obesity have not been matched by effective medical responses.

Like hypertension, hyperlipidemia, and sleep apnea, obesity can be legitimately viewed as a chronic disease, requiring long-term treatments and sustained effort. The current challenge posed by obesity management is partly the dearth of effective clinical treatments. As with earlier days of treatment for hypertension or cholesterol, increasingly effective treatments are coming, but individual treatment successes are possible.

Efforts to manage obesity are further challenged by the necessary reliance of available treatments on complementary behavior modifications. To the extent that obesity-related behaviors can be effectively treated, success often occurs in the context of a specialized program. Because efforts to change behavior require considerable interaction time, the primary care physician is disadvantaged in efforts to address this condition. Limitations by payers on the time that can be spent, either by the primary care physician or by associated practitioners, to address these behavioral concerns mean that the practitioner is under great pressure to do much in a short time. Furthermore, many payers will not compensate for such treatments, and the practitioner, although often sympathetic with the goal of improved body weight, feels unable to meet the patient's needs.

Submitted 15 March 2000.

From Minnesota Obesity Center, Veterans Affairs Medical Center, Minnesota. Address reprint requests to Charles J. Billington, MD, Minnesota Obesity Center, Veterans Affairs Medical Center, One Veterans Drive, Delivery Code $111 \mathrm{P}$, Minneapolis, MN 55417.
The great challenge of translating obesity treatment knowledge into primary care practice is addressed in the current study by Logue and colleagues on obesity management in primary care. ${ }^{2}$ The study separately considered six individual behaviors that could be logically modified to improve body weight, such as regular exercise and reducing portion size. Obese patients were assessed with respect to their stage-of-change status for each behavior. Patients as a group were distributed throughout the spectrum of change behavior, from just beginning to think about it (precontemplation) to action on a goal behavior. Interestingly, patients were not necessarily coherent in their choices about the six behaviors, so action in one dimension might well be paired with only consideration in another. There was an indication that patients who had progressed toward active engagement with a behavior, notably exercise-related behaviors, were more likely to have a lower body mass index than those who were considering such changes.

These findings are in accord with the recommendations of many. The Practical Guide to the Clinical Guidelines on the Identification, Evaluation, and Treatment of Overweight and Obesity in Adults ${ }^{3}$ recommends that the primary care physician determine an individual's readiness to change before attempting behavior modification. This plan focuses resources on those most likely to benefit. The current study, although cause and effect cannot be attributed, suggests that those more ready to change are doing better with their weight. Individual primary care practitioners can use this information to direct their efforts. Given the current state of knowledge, it would be logical to pick one at a time from the six weight-related behaviors and attempt to help the patient move toward the action end of the stages-of-change spectrum. Reducing a behavioral weight management program into bits that can be used during short primary care encounters might make good sense. Studies of methods that might be used to translate such a program into primary care practice, and the practical experience 
of such programs, would appear to be of high priority.

Charles J. Billington, MD

Minnsota Obesity Center Veterans Affairs Medical Center

Minneapolis

\section{References}

1. Clinical guidelines on the identification, evaluation, and treatment of overweight and obesity in adults. National Institutes of Health, National Heart, Lung and Blood Institute. Obesity Res 1998;6(Suppl 2): 51S-209S.

2. Logue E, Sutton K, Jarjoura D, Smucker W. Obesity management in primary care: assessment of readiness to change among 284 family practice patients. J Am Board Fam Pract 2000;13:164-171.

3. Practical guide to the clinical guidelines on the identification, evaluation, and treatment of overweight and obesity in adults. National Institutes of Health, National Heart, Lung and Blood Institute. Washington, DC: US Government Printing Office, in press. 